Bernhard Laufer*, Paul D. Docherty, Sabine Krueger-Ziolek, Fabian Hoeflinger, Leonhard Reindl, Knut Moeller

\title{
An alternative way to measure total lung capacity: a pilot study
}

\begin{abstract}
Total lung capacity is usually measured by a body plethysmograph or helium dilution methods. In this study an alternative approach to obtain total lung capacity of spontaneous breathing subjects is introduced. The device utilises an optoelectronic plethysmograph and a small tube, which measures mouth pressure and allows total lung capacity to be obtained, which differs less than $0.4 \mathrm{~L}$ from the total lung capacity of the body plethysmograph. The method shows potential to be a less burdensome method to estimate total lung capacity determination than the body plethysmograph.
\end{abstract}

Keywords: Total lung capacity, optoelectronic plethysmography, respiratory parameters

https://doi.org/10.1515/cdbme-2020-3061

\section{Introduction}

Respiratory parameters are important for analysis and treatment of critically ill patients and have been the focus of various measurement systems to obtain the desired parameters $[1,2]$. This study will consider two of these systems. One method is spirometry, which measures the airflow through a facemask or mouthpiece. The flow of air through the mouth allows measurement of respiratory parameters, such as tidal volume, vital capacity (VC) and a couple of dynamic respiratory parameters (e.g. forced vital capacity FVC and forced expiratory volume in 1 sec FEV1). Due to high accuracy and moderate financial costs, spirometers are the research for some decades. This has led to the development of

gold standard and the most widespread used method all around the world [3].

The other system considered is the optoelectronic plethysmography (OEP), which is not often utilised in a clinical setting [4]. OEP is a solution for specific applications, such as respiratory monitoring of neonates or infants, and is based on an expensive motion capture system which can measure thoracic surface motions precisely. These motions are subsequently used to calculate respiration induced volume changes of the upper body.

However, if the total lung capacity TLC is of interest, both spirometry and OEP fail. Even though there have been some preliminary attempts to generate model based estimates for TLC from OEP data [5]. Hence, to determine TLC, sophisticated measurement systems have to be utilized, such as body plethysmography or helium dilution methods $[6,7]$. Despite the costs and the large dimension of a body plethysmograph, this measurement system is the gold standard for TLC determination and can be found in most pulmonary clinic services, while helium dilution methods are less frequently used.

In this feasibility study, a new method to measure the total lung capacity is introduced. This method is based on an OEP and a small tube with implemented pressure sensor to calculate TLC. The ultimate goal of this research stream to substitute the OEP with a wearable smart-shirt, which delivers respiration induced volume data. Hence, the augmented OEP approach could be able to obtain TLC with much lower burdens of cost, equipment size, and immobility than the gold standard body plethysmograph.

\section{Methods}

*Corresponding author: Bernhard Laufer: Institute of Technical Medicine (ITeM), Furtwangen University, Villingen-Schwenningen, Germany, b.laufer@hs-furtwangen.de

Sabine Krueger-Ziolek, Knut Moeller: ITeM, Furtwangen University, Villingen-Schwenningen, Germany

Paul D. Docherty: University of Canterbury, Christchurch, New Zealand

Fabian Hoeflinger, Leonhard Reindl: University of Freiburg, Freiburg, Germany

\subsection{Measurement system}

The introduced TLC-device is a small tube with an inspiration valve at the end and an integrated digital pressure sensor (BMP280, Bosch Sensortec GmbH, Reutlingen, Germany) inside the tube (see Error! Reference source not found.). 
The inhalation valve in the device only allows one-directional airflow - the subject can inhale but not exhale. To ensure the subject's safety, the tube is combined with a bacterial filter. The pressure sensor was connected to an inexpensive Arduino MKR Wifi 1010 board (ARDUINO.CC and Arduino IDE Version 1.8.8) with SAMD21 controller. The data were transferred via serial interface to MATLAB (R2019a, The MathWorks, Natick, USA).

To measure volume changes of the upper body during breathing, an OEP system was used, which was based on a motion capture system (see Error! Reference source not found.). This system can measure the spatial positions of the deployed 102 reflecting markers (fixed on a compression shirt) accurately. The VICON motion capture-system (Bonita, VICON, Denver, CO) consisted of 9 infrared cameras (VICON Bonita B10, Firmware Version 404). The VICON Nexus Software (Version 1.8.5.6 1009h, Vicon Motion Systems Ltd.) was used to transfer the spatial positions of each marker to MATLAB, where all further calculations were done.



Figure 1: Picture of the TLC-device, bacterial filter, tube, pressure sensor and Arduino board

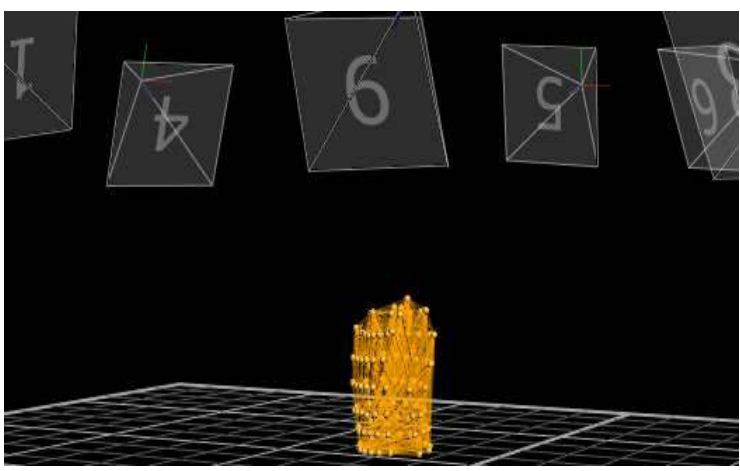

Figure 2: Schematic sketch of the OEP system - 9 infrared cameras and the shirt with 102 motion capture markers.

As reference system for TLC determination a body plethysmograph (PowerCube ${ }^{\circledR}$ Body+, Ganshorn Medizin Electronic GmbH, Niederlauer, Germany) and its LFX software (Version 1.8.1) was used.

\subsection{Participants / manoeuvre}

This feasibility study was undertaken with 3 lung healthy subjects. Detailed data from the subjects can be found in Table 1. Written informed consent was collected from all subjects.

Table 1. Participants.

\begin{tabular}{llllll}
\hline \multirow{2}{*}{ Subject } & $\begin{array}{l}\text { Height } \\
{[\mathbf{m}]}\end{array}$ & $\begin{array}{l}\text { Weight } \\
{[\mathbf{k g}]}\end{array}$ & $\begin{array}{l}\mathbf{B M I} \\
{\left[\mathbf{k g} / \mathbf{m}^{2}\right]}\end{array}$ & $\begin{array}{l}\text { Age } \\
{[\text { years] }}\end{array}$ & Gender \\
\hline 1 & 1.82 & 70 & 21.1 & 18 & male \\
2 & 1.72 & 63 & 21.3 & 19 & female \\
3 & 1.75 & 62 & 20.2 & 22 & male \\
\hline
\end{tabular}

After a reference measurement of the TLC by means of the body plethysmograph, the subject, wearing the compression shirt with markers, breathed normally for about 30 seconds. Afterwards, at start of inhalation the subject used the TLC-device to inhale maximally through the inspiration valve and hold the air for more than 2 seconds. This "breath holding" was done to avoid BTPS (body temperature, pressure, saturated with water vapor) corrections, which were usually necessary to take the temperature and humidity differences between inspired ambient air and the air inside the lung into account [8].

The subsequent exhalation was blocked by the inspiration valve and thus, while the subject tried to exhale, the pressure inside the TLC-device increased. The subjects were advised not to "blow up" the cheeks during this manoeuvre as this would distort the measurement. 
After some seconds of "exhalation", or more specifically pressure generation, the subject removed the TLC device and continued the normal spontaneous breathing.

\subsection{Data Processing}

The Boyle Mariotte law states that in a closed system with constant temperature the product of pressure $p$ and volume $V$ is constant $(p \cdot V=$ const $)$.

By using the TLC-device, at the end of maximal inhalation, the pressure inside lungs and device was atmospheric pressure $p_{\text {atm }}$ while the lungs of the subject were maximally filled. The following attempted of "exhalation" increased the pressure because the valve was closed. This pressure $p_{\text {mouth }}$ increase was associated with a volume reduction inside the lung by $\Delta V_{\text {lungs }}$ and thus, as well as with a volume reduction of the human upper body $\Delta V_{\text {UpperBody }}$.

Assuming, that the change in volume of the human upper body roughly equals the change in volume of the lung and airways, the reduced volume $V_{\text {reduced }}$ is given by

$$
V_{\text {reduced }}=V_{T L C}-\Delta V_{\text {lungs }} \approx V_{T L C}-\Delta V_{\text {UpperBody }}
$$

where: $V_{T L C}$ is the total lung capacity.

Therefore, based on the Boyle Mariotte law the total lung capacity $V_{T L C}$ can be calculated by:

$$
p_{\text {atm }} \cdot V_{T L C}=p_{\text {mouth }} \cdot V_{\text {reduced }}
$$

which, by taking Equation 1 into account, results in:

$$
V_{T L C} \approx \Delta V_{\text {UpperBody }} \cdot p_{\text {mouth }} \cdot\left(p_{\text {mouth }}-p_{\text {atm }}\right)^{-1}-V_{d}
$$

where: $p_{\text {mouth }}$ is the pressure inside the TLC-device and $p_{\text {atm }}$ is the atmospheric pressure. $V_{d} \approx 200 \mathrm{ml}$ is a volume correction term, which was introduced to correct the error caused by the additional dead space inside the bacterial filter, tube, and mouth (see Figure 1). The minimum of $V_{T L C}$ curve was considered to be the total lung capacity as this allows the removal of dynamics effects, the effects of possible compression of non-pulmonary tissues in the thorax.

\section{Results}

Thoracic gas volume TGV manoeuvres and a subsequent Tiffeneau manoeuvre yield to the total lung capacity $V_{T L C, b o d y}$ of the subject via the body plethysmograph.

Fig. 3 illustrates the volume of the upper body measured by the OEP, the mouth pressure obtained via the TLC-device and the TLC curve calculated by means of Equation 3.
Table 2 lists the results for TLC, obtained by the TLCdevice and the body plethysmograph.

Table 2 Results for TLC

\begin{tabular}{lll}
\hline subject & $V_{\text {TLC-device }}[L]$ & $V_{T L C, \text { body }}[L]$ \\
\hline 1 & 7.7 & 7.6 \\
2 & 7.2 & 7.0 \\
3 & 8.1 & 7.7 \\
\hline
\end{tabular}

Figure 3: Volume gained by the OEP (top), mouth pressure obtained by the TLC-device (middle) and the resulting total lung capacity $V_{T L C}($ down), based on the data of subject 2. After inspiration, breath holding is done, and the orange highlighted phase is the TLC measurement.
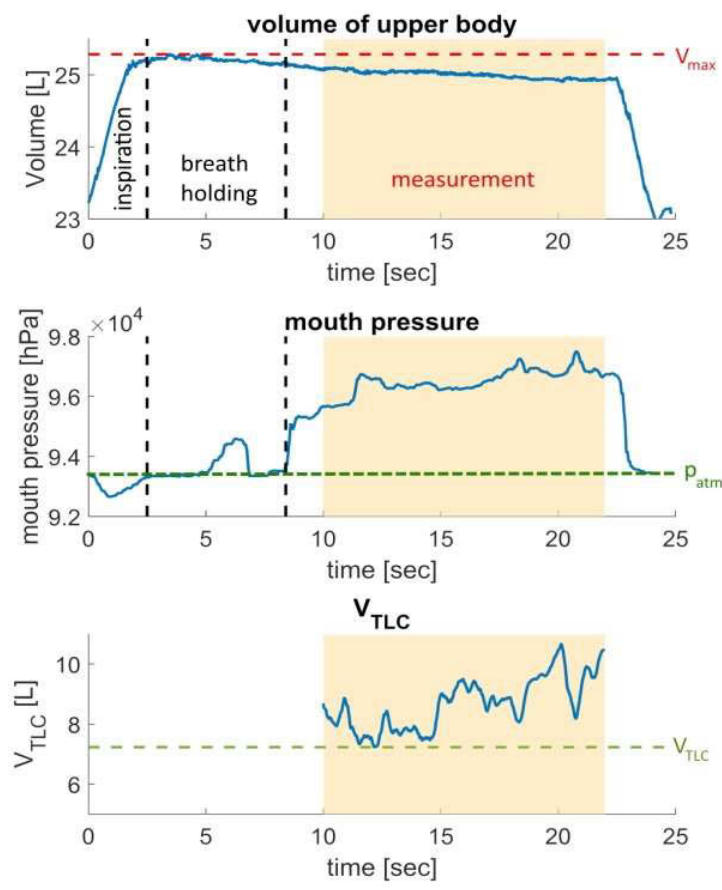


\section{Discussion}

Normally, total lung capacity is measured by a body plethysmograph or by means of a helium dilution device. However, the gold standard body plethysmograph shows high variances for TLC measurement [7], and is a cumbersome and expensive process to perform.

The introduced device supports measurement systems, such as the OEP or smart shirts in future, measuring the respiration induced changes of volume inside the upper body, to obtain beside other respiratory parameters, the total lung capacity. In this pilot study, the TLC-device showed that the obtained TLCs are in the range of the TLC values of the body plethysmograph and that the measurement setup (OEP + TLC device) is able to measure TLC values. To evaluate these results and the robustness of the measurement, more measurements with more participants should be undertaken.

A body plethysmography measurement requires a BTPS correction, which takes the different temperatures and water saturations of the surrounding air and the air inside the lungs into account. By breath holding for more than 2 seconds during the measurement via the TLC device a BTPS correction is not required, because it can be assumed that after that time, the air inside the lung is adjusted to body conditions. During the subsequent phase of measurement, which is used for TLC calculation, the air has nearly constant condition $\left(37^{\circ} \mathrm{C}\right.$ body temperature and $100 \%$ saturation with water) and the exhalation inside the small device, the condition of the expired air does not change significantly.

Before and after the measurement phase (orange highlighted in Figure 3) the mouth pressure is very close to atmospheric pressure. Since the term $\left(p_{\text {mouth }}-p_{\text {atm }}\right)$ is in the denominator of Equation 2, small aberrations in measured pressure can result in large apparent fluctuations in the calculated $V_{T C L}$ value.

The assumption that the volume change of the upper body equals the volume change inside the lungs leads to an overestimation of the total lung capacity obtained by the TLC device. This systematic error is caused by the additional pressure dependent volume change of the tissue inside the upper body, which results that $\Delta V_{\text {UpperBody }}$ is slightly higher than $\Delta V_{\text {lungs. }}$. Therefore, among the various values that are obtainable from a profile, this research selected the minimum value of the $V_{T C L}$ profile. This selection should be evaluated and checked for robustness for a higher number of subjects. Additionally, a correction term $V_{d}$ was subtracted from the TLC curve, which is the dead space of the TLC-device.

Using the OEP, the volume measurement is restricted to the upper body and therefore, if the subjects allow their cheeks to become puffed up with air during the measurement; significant errors are likely to occur. In contrast to the measurement in the body plethysmograph, the air in the cheeks is not considered in Equation 3 and assumptions of the conditions of the Boyle Mariotte law are invalidated.

The TLC-device together with a Smart-Shirt would provide a very cost-effective method to measure the TLC and would make this important diagnostic parameter accessible for a wide range of medical practices and clinics from intensive care wards to general wards and elderly care.

Future work should include a subsequent evaluation of the TLC-device with a higher number of participants. Furthermore, this study was based on an OEP system to measure the volume change of the upper body; further developments might try to employ a wearable system, such as a smart shirt, to obtain the desired volume change of the upper body. If this could be achieved, a further study with elderly or patients with lung diseases should follow, where the pressure change is expected to be small.

\section{Conclusion}

This study showed that the augmented OEP approach can be used to obtain the total lung capacity. Further developments using a smart shirt to determine volume changes instead of an OEP combined with the introduced TLC-device have the potential to be a non-invasive, cost effective and wearable alternative for TLC determination.

\section{Acknowledgement and Author Statement}

Research funding: This research was partly supported by the German Federal Ministry of Education and Research (MOVE, Grant 13FH628IX6) and H2020 MSCA Rise (\#872488DCPM).

Conflict of interest: Authors state no conflict of interest. Informed consent: Informed consent has been obtained from all individuals included in this study. Ethical approval: The research related to human use complies with all the relevant national regulations, institutional policies and was performed in accordance with the tenets of the Helsinki Declaration, and has been approved by the authors' institutional review board or equivalent committee.

\section{References}

[1] Wanger J, Clausen JL, Coates A, Pedersen OF, Brusasco V, Burgos $\mathrm{F}$, Casaburi $\mathrm{R}$, Crapo $\mathrm{R}$, Enright $\mathrm{P}$, et al. Standardisation of the measurement of lung volumes. European Respiratory Journal 2005; 26 (3): 511. 
[2] Lutfi MF. The physiological basis and clinical significance of lung volume measurements. Multidisciplinary respiratory medicine 2017; 12: 3-3.

[3] Miller MR, Hankinson J, Brusasco V, Burgos F, Casaburi R, Coates A, Crapo R, Enright P, van der Grinten CP, et al. Standardisation of spirometry. Eur Respir J 2005; 26 (2): 31938.

[4] Massaroni C, Carraro E, Vianello A, Miccinilli S, Morrone M, Levai IK, Schena E, Saccomandi $\mathrm{P}$, Sterzi $\mathrm{S}$, et al. Optoelectronic Plethysmography in Clinical Practice and Research: A Review. Respiration 2017; 93 (5): 339-354.

[5] Dellacà RL, Aliverti A, Pelosi P, Carlesso E, Chiumello D, Pedotti A, Gattinoni L. Estimation of end-expiratory lung volume variations by optoelectronic plethysmography. Critical Care Medicine 2001; 29 (9): 1807-1811.

[6] Criée CP, Sorichter S, Smith HJ, Kardos P, Merget R, Heise $D$, Berdel D, Köhler D, Magnussen $H$, et al. Body plethysmography - Its principles and clinical use. Respiratory Medicine 2011; 105 (7): 959-971.

[7] Tang $Y$, Zhang $M$, Feng $Y$, Liang $B$. The measurement of lung volumes using body plethysmography and helium dilution methods in COPD patients: a correlation and diagnosis analysis. Scientific reports 2016; 6: 37550-37550.

[8] Vooren PH. A Nomographic Ruler for Body Temperature, Pressure, Saturated with Water (BTPS) Correction. American Review of Respiratory Disease 1967; 96 (2): 324-325. 